\title{
HOW TO STUDY SCIENTIFIC EXPLANATION?
}

Erik Weber, Leen De Vreese \& Jeroen Van Bouwel

Centre for Logic and Philosophy of Science

Ghent University (UGent)

Blandijnberg 2

B-9000 Gent

Belgium

Erik.Weber@UGent.be

Leen.DeVreese@UGent.be

Jeroen.VanBouwel@UGent.be

\begin{abstract}
This paper investigates the working-method of three important philosophers of explanation: Carl Hempel, Philip Kitcher and Wesley Salmon. We argue that they do three things: (i) construct an explication in the sense of Carnap, which then is used as a tool to make (ii) descriptive and (iii) normative claims about the explanatory practice of scientists. We also show that they did well with respect to (i), but that they failed to give arguments for their descriptive and normative claims. We think it is the responsibility of current philosophers of explanation to go on where Hempel, Kitcher and Salmon failed. However, we should go on in a clever way. We call this clever way the "pragmatic approach to scientific explanation." We clarify what this approach consists in and defend it.
\end{abstract}

\section{Keywords}

Carnap, explanation, explication, Hempel, Kitcher, Salmon.

This text is the English version of a paper that has been published in Dutch:

Erik Weber, Leen De Vreese \& Jeroen Van Bouwel, 'Hoe moeten we wetenschappelijke verklaring bestuderen?', in Tijdschrift voor Filosofie 78 (2016), pp. 489-524. 


\section{HOW TO STUDY SCIENTIFIC EXPLANATION?}

\section{Introduction}

In the first part of this paper we investigate the working-method of three important philosophers of explanation: Carl Hempel, Philip Kitcher and Wesley Salmon. We argue that they do three things: (i) construct an explication in the sense of Rudolf Carnap, which then is used as a tool to make (ii) descriptive and (iii) normative claims about the explanatory practice of scientists. In Section $2-$ which has a preliminary character - we present Carnap's view on what the task of explication is, on the requirements it has to satisfy and on its function. In Section 3 we show that Carl Hempel develops an explication of the concept of explanation and makes descriptive and normative claims with it. We also show that he fails to provide convincing arguments for these claims. In Section 4 and 5 we show that Philip Kitcher and Wesley Salmon had a similar working-method in their philosophical analysis of scientific explanation and failed at the same stage as Hempel: the arguments for the descriptive and normative claims are missing.

Though a bit more than half of this paper is historical, our ultimate aim is metaphilosophical. As our title suggests, we want to present and defend a metaphilosophical view on how to study scientific explanation. We call this view the "pragmatic approach to scientific explanation". In Sections 6 and 7 we clarify what this approach consists in and provide some theoretical arguments for it. In Section 8 we present a case study in which the approach is implemented. This case study clarifies the approach and provides a further argument for it.

The two parts of the paper are of course connected: the diagnosis of what brought Hempel, Kitcher and Salmon into trouble helps us to avoid the same problems. So we think there are important lessons to be drawn from a reflection on the work of the three philosophers.

For readers who are not very well acquainted with the philosophical literature on scientific explanation, we have added an appendix containing a brief overview of Hempel's DN-model and the problems that other philosophers saw in it.

\section{Rudolf Carnap on explication}

Carnap devotes Chapter 1 of his Logical Foundations of Probability to the notion of explication. The following quote summarises his view:

According to these considerations, the task of explication may be characterized as follows. If a concept is given as explicandum, the task consists in finding another concept as its explicatum which fulfils the following requirements to a sufficient degree.

1. the explicatum is to be similar to the explicandum in such a way that, in most cases in which the explicandum has so far been used, the explicatum can be used; however, close similarity is not required, and considerable differences are permitted.

2. The characterization of the explicatum, that is, the rules of its use (for instance, in the form of a definition), is to be given in an exact form, so as to introduce the explicatum into a well-connected system of scientific concepts.

3. The explicatum is to be a fruitful concept, that is, useful for the formulation of many universal statements (empirical laws in the case of a nonlogical concept, logical theorems in the case of a logical concept).

4. The explicatum should be as simple as possible; this means as simple as the more important 
requirements (1), (2), (3) permit. $(1950$, p. 7)

According to Carnap, scientists often make explications. One of his examples is the replacement of the concept of Fish by the concept of Piscis:

That the explicandum Fish has been replaced by the explicatum Piscis does not mean that the former term can always be replaced by the latter; because of the difference in meaning just mentioned, this is obviously not the case. The former concept has been succeeded by the latter in this sense: the former is no longer necessary in scientific talk; most of what previously was said with the former can now be said with the help of the latter (though often in a different form, not by simple replacement).

... [T] he concept Piscis promised to be much more fruitful than any concept more similar to Fish. A scientific concept is the more fruitful the more it can be brought into connection with other concepts on the basis of observed facts; in other words, the more it can be used for the formulation of laws. The zoölogists found that the animals to which the concept Fish applies, that is, those living in water, have by far not as many other properties in common as the animals which live in water, are cold-blooded vertebrates, and have gills throughout life. Hence, the concept Piscis defined by these latter properties allows more general statements than any concept defined so as to be more similar to Fish; and this is what makes the concept Piscis more fruitful. (1950, p. 6)

His second scientific example is Temperature as explicatum of Warmer (see 1950, p. 12). In both cases, the explicatum allows us to formulate more empirical laws than the explicandum, and that is why they are fruitful.

Carnap claims that also philosophers often make explications. His aim in his 1950 book is to develop a good explication of the concepts of probability and (degree of) confirmation. Before we turn to the use of the concept of explication by philosophers of explanation, it is important to note that the fruitfulness of philosophical explications may be of a different nature than the fruitfulness of scientific ones. It is possible that the fruitfulness of philosophical explications lies in the formulation of empirical generalisations (like scientific explications). But a serious option to consider is that their fruitfulness lies in that they allow us to offer clear guidelines for scientists (i.e. we formulate norms with them, not empirical generalisations). As we will see, Hempel, Kitcher and Salmon try to exploit both these possibilities.

We have presented Carnap's idea of explication here in some detail because we wanted to draw attention to the fact that for him explication is not an aim in itself, it is a means for something else. The Piscis example illustrates this very well: first you develop the new concept, then you formulate new hypotheses with it, and then you collect data to test these hypotheses. Analogously, philosophical explications are not an aim in se: we have to put them to use. Hempel, Kitcher and Salmon understood that: they develop an explicatum and then use it.

\section{Carl Hempel's working-method}

The first stage: explication

According to the DN model and IS model, we explain particular facts by subsuming them under a law. The law then, so to speak, "covers" the fact to be explained. For this reason Hempel's views on explanation are known as the covering law model. 
It is clear that Hempel sees his covering law model as an explication in the sense of Carnap. At the end of the long essay 'Aspects of Scientific Explanation' he writes:

This construal, which has been set forth in detail in the preceding sections, does not claim simply to be descriptive of the explanations actually offered in empirical science; for - to mention but one reason - there is no sufficiently clear generally accepted understanding as to what counts as a scientific explanation. The construal here set forth is, rather, in the nature of an explication, which is intended to replace a familiar but vague and ambiguous notion by a more precisely characterized and systematically fruitful and illuminating one. Actually, our explicatory analysis has not even led to a full definition of a precise "explicatum"-concept of scientific explanation; it purports only to make explicit some especially important aspects of such a concept.

Like any other explication, the construal here put forward has to be justified by appropriate arguments. In our case, these have to show that the proposed construal does justice to such accounts as are generally agreed to be instances of scientific explanation, and that it affords a basis for a systematically fruitful logical and methodological analysis of the explanatory procedures used in empirical science. (1965, pp. 488-489)

If we compare this with Carnap's criteria, we notice the following:

(1) Two of Carnap's criteria are explicitly mentioned: fruitfulness and exactness.

(2) Hempel does not mention similarity to the explicandum explicitly. However, proposals should do justice to what are generally agreed as instances of explanation (cf. the last sentence of the quote). "Doing justice to" is vague, but we think it can safely be assumed to correspond to Carnap's similarity: no exact match, but not too much dissimilarity.

(3) Simplicity is not mentioned. Is it self-evident for Hempel that philosophers should be as simple as possible, or does Hempel think that simplicity is not a cognitive value for philosophers? Given how he proceeds, we bet on the first answer.

Based on this comparison, it is reasonable to assume that Hempel's idea of explication is the same as Carnap's.

\section{Descriptive and normative claims}

Hempel has a clear view on the fruitfulness of philosophical models of explanation. This view is expressed in the following quote:

As is made clear by our previous discussions, these models are not meant to describe how working scientists actually formulate their explanatory accounts. Their purpose is rather to indicate in reasonably precise terms the logical structure and the rationale of various ways in which empirical science answers explanation-seeking why-questions. (1965, p. 412)

The construal here broadly summarized is not, of course, susceptible to strict "proof"; its soundness has to be judged by the light it can shed on the rationale and force of explanatory accounts offered in different branches of empirical science. (1965, p. 425)

Hempel suggests here that his models give us insight in the logical structure of explanation (What do they look like?) and the rationale of explanations (Why do scientists construct them? Why are they valuable?). How this second aspect works can be seen in the following quote:

Thus a D-N Explanation answers the question 'Why did the explanandum-phenomenon occur?' by showing that the phenomenon resulted from certain particular circumstances, specified in 
$C_{1}, C_{2}, \ldots, C_{k}$, in accordance with the laws $L_{1}, L_{2}, \ldots, L_{\mathrm{r}}$. By pointing this out, the argument shows that, given the particular circumstances and the laws in question, the occurrence of the phenomenon was to be expected; and it is in this sense that the explanation enables us to understand why the phenomenon occurred. (p. 337).

In other words: explanations provide understanding and the covering law models - according to Hempel - provide insight into what understanding really is.

In claiming that his models give insight in the logical structure of explanations and in connecting DN and IS explanation with expectability and understanding, Hempel puts forward the following descriptive hypothesis:

All scientists who have understanding as an aim really seek DN or IS explanations, so that the phenomenon they want to understand becomes expectable.

This hypothesis cannot be formulated without the explicatum. In this way, the explicatum is fruitful. In order to avoid misunderstanding of the descriptive claim, it is important to stress that it is not a claim about what scientists or laymen call explanations. It is a claim about the intentions of scientists, about the kind of understanding they are after.

In the quote above Hempel not only mentions "rationale" but also "force". One of the insights we get from his models - in his view - is that non-causal explanations can be as good as causal explanations: they can have the same explanatory force. Therefore, there is no reason to demand that a good scientific explanation should be causal. Hempel states that it is unclear "what reason there would be denying the status of explanation to all accounts invoking occurrences that temporally succeed the event to be explained" (pp. 353-354). In other words: a DN or IS explanation is sufficient for providing understanding. They are also necessary for Hempel, as the following quote illustrates:

I think that all adequate scientific explanations and their everyday counterparts claim or presuppose implicitly the deductive or inductive subsumability of whatever is to be explained under general laws or theoretical principles. In the explanation of an individual occurrence, those general nomic principles are required to connect the explanandum event with other particulars [.] (p. 424-425)

This view can be summarised in the following normative claim:

All scientists that are engaged in trying to understand the world should construct DN or IS explanations (and not necessarily something more specific, such as DN explanations citing causes).

This guideline cannot be formulated without the concepts of DN and IS explanation. Again, this explicatum is fruitful: we can formulate a guideline that we cannot formulate without it.

\section{Hempel's failures}

Let us now have a look at the problems. A first problem is that Hempel does not provide arguments for his descriptive claim. He does not provide a database with records of relevant opinions of a large representative sample of scientists from all disciplines all over the world (i.e. their opinions on what kind of understanding is important). However, that is what he should have done if he wanted to build a convincing argument for his empirical claim. If we compare what he does with what Carnap has in 
mind with "Piscis" and other scientific explicata, Hempel resembles a scientist who develops a nice explicatum concept, formulates an interesting hypothesis with it and then stops instead of trying to collect empirical evidence. Hempel stops where the real challenge starts: gathering evidence for his hypothesis by systematically investigating the opinions of a large representative sample of scientists (this could be done by interviewing them or analysing their writings).

Our criticism of Hempel is similar to the complaints of experimental philosophers about appeals to intuition in analytic philosophy: claims about what laymen would say about a particular case are part of the argument for a philosophical theory, but traditional philosophers do not actually ask people what they think. More generally traditional philosophy (which experimental philosophers call armchair philosophy) does not use the standard empirical methods of the behavioural and social sciences when they make descriptive claims about human attitudes, opinions, behaviour, etc. (see Knobe 2004 for a concise statement of what experimental philosophy is).

The second problem is that Hempel does not give arguments for his normative claim. Why, for instance, are causal explanations not interesting in a special sense (i.e. in a sense different from the general one in which the set of DN-explanations, of which they are a subset, are interesting)? Again, he stops where the real challenge begins.

\section{Philip Kitcher's working-method}

\section{Kitcher versus Hempel}

In his paper 'Explanatory Unification' Philip Kitcher writes:

Why should we want an account of explanation? Two reasons present themselves. Firstly, we would like to understand and to evaluate the popular claim that the natural sciences do not merely pile up unrelated items of knowledge of more or less practical significance, but that they increase our understanding of the world. A theory of explanation should show us how scientific explanation advances our understanding. ... Secondly, an account of explanation ought to enable us to comprehend and to arbitrate disputes in past and present science. Embryonic theories are often defended by appeal to their explanatory power. A theory of explanation should enable us to judge the adequacy of the defense. $(1981$, p. 508)

The first reason Kitcher mentions is similar to what Hempel calls "rationale". However, Kitcher claims that Hempel has it all wrong: understanding consists in unification, not in expectability. They have a common aim, but Kitcher thinks that Hempel has failed. Kitcher does not mention the concept of explication explicitly, but his model of explanation satisfies Carnap's criteria: precise definition, similarity and fruitfulness (Kitcher formulates descriptive and normative hypotheses with it, see below).

On the descriptive side, Kitcher puts forward two claims. The first is a negative one, a claim against Hempel:

Scientists who have understanding as an aim often seek something more specific than DN explanations. So Hempel's descriptive hypothesis is false.

This claim can be found at several places in Kitcher's work. For instance, in commenting on Hempel's covering law model he writes:

Many derivations which are intuitively nonexplanatory meet the conditions of the model. 
(1981, p. 508)

The derivations which Kitcher has in mind are all the counterexamples which several philosophers have launched against Hempel (cf. our Appendix). In order to grasp Kitcher's critique of Hempel, it is important to remember that they are not interested in what scientists and other people call explanations. They are interested in what understanding is. According to Kitcher, if you would present a scientist with the two derivations of the flagpole example (see Appendix) then this scientist would say that the derivation of the length of the shadow from the height of the flagpole provides understanding, while the other derivation does not. So the first derivation would be judged as scientifically more interesting. Kitcher is convinced that scientists would judge similarly in other cases (e.g. the pendulum) and concludes that expectability is not the kind of understanding scientists are after. According to Hempel, scientists would judge that both derivations are equally interesting (the derivation of the height from the flagpole provides as much understanding as the other derivation). Similarly for the other cases such as the pendulum. Hempel concludes that expectability is the kind of understanding that scientists are after.

The problem with Kitcher's line of reasoning is the same as with Hempel's: he speculates about what scientists would answer when presented with a set of derivations and a corresponding question. He did not actually interview a representative sample of scientists. The result is that the dispute cannot be settled: both Hempel and Kitcher claim that if one would consult scientists, that would result in evidence supporting their hypotheses. They cannot both be right.

Kitcher's positive descriptive claim

Kitcher also puts forward a positive descriptive claim:

All scientists who have understanding as an aim really seek $U$ explanations, so that the phenomena they want to understand become more unified.

"U explanation" stands for "unification explanation". ${ }^{1}$ An underlying idea of Kitcher is that, while all explanations are arguments, the converse is not true. He uses argument patterns to distinguish explanations from non-explanatory arguments. For an individual with knowledge $\mathrm{K}$, an argument $\mathrm{A}$ can only be an explanation if it is acceptable relative to $K$ (i.e. if the premises of $A$ are members of $K$ ). But not all acceptable arguments are explanations: an acceptable argument is an explanation if and only if it instantiates an argument pattern that belongs to a privileged set of argument patterns. This set of argument patterns is privileged because it has a higher unifying power with respect to $K$ than any other conceivable set of argument patterns. The unifying power of a set of argument patterns is determined by four factors: (i) it varies directly with the number of accepted sentences (i.e. the number of elements of $\mathrm{K}$ ) that can be derived by means of acceptable arguments that instantiate a pattern in the set; (ii) it varies conversely with the number of patterns in the set; (iii) it varies directly with the stringency of its members; and (iv) it varies directly with the degree of similarity of its members.

Here is a quote from Kitcher in which the positive descriptive claim is clearly present:

Science advances our understanding of nature by showing us how to derive descriptions of many phenomena, using the same patterns of derivation again and again, and, in demonstrating this, it teaches us how to reduce the number of types of facts we have to accept

\footnotetext{
${ }^{1}$ There is no standard letter code, comparable to "DN" or "IS" for Hempel's views, to denote explanations in the sense of Kitcher. Because his account is unificationist, we choose the letter " $U$ ".
} 
as ultimate (or brute). (1989, p. 432; italics in original)

In Kitcher's work on explanation no evidence for this second, positive claim can be found. He has not surveyed a large sample of scientists of various disciplines, in order to investigate what their views on understanding are. He clarifies the meaning of his claim (by developing his account of unification) but - like Hempel - he stops where the real challenge starts: gathering empirical evidence for the descriptive hypothesis.

Kitcher's normative claim

Kitcher also puts forward a normative claim:

All scientists that are engaged in trying to understand the world should construct $U$ explanations (nothing more, nothing less).

This is a strong claim. On the one hand, it excludes the formats according to which explanations are no arguments, e.g. those proposed in Cartwright 1983, Humphreys 1989 or Salmon 1984. A U explanation is always an argument, so what these non-argument views propose as explanation formats is "less" than a $U$ explanation and thus not good enough. On the other hand, it implies that causal information is superfluous: causal explanations are "more" than $U$ explanations, and we should not aim at this "more". For Kitcher, causal information is only useful to the extent that it promotes unification. It is not an aim in itself. Here is a quote from Kitcher in which he clearly expresses a normative aim:

The most general problem of scientific explanation is to determine the conditions which must be met if science is to be used in answering an explanation-seeking question $Q$. I shall restrict my attention to explanation-seeking why-questions, and I shall attempt to determine the conditions under which an argument whose conclusion is $S$ can be used to answer the question "Why is it the case that $S$ ?" (1981, p. 510; emphasis added)

Kitcher does not argue for this normative claim either. Again, he stops where the real challenge starts.

\section{Wesley Salmon's working method}

\section{Salmon's descriptive claim}

In Chapter 1 of Scientific Explanation and the Causal Structure of the World, Wesley Salmon explicitly puts himself in the tradition of explication:

Many philosophical studies, including the one to which this book is devoted, aim at providing reasonably precise explications of fundamental concepts [.] (1984, p. 4)

Like Hempel and Kitcher, he claims that his explication of the concept of scientific explanation provides insight into what understanding is:

Our aim is to understand scientific understanding. We secure scientific understanding by providing scientific explanations; thus our main concern will be with the nature of scientific explanation. (1984, p. ix) 
Scientific explanations can be given for such particular occurrences as the appearance of Halley's comet in 1759 or the crash of a DC-10 jet airliner in Chicago in 1979, as well as such general features of the world as the nearly elliptical orbits of planets or the electrical conductivity of copper. The chief aim of this book is to try to discover just what scientific understanding of this sort consists in. (1984, p. 3, emphasis added)

His ambitions are quite high. He hopes to have achieved this aim for all sciences except quantum mechanics:

It is my hope that the causal theory of scientific explanation outlined in this book is reasonably adequate for the characterization of explanation in most scientific contexts - in the physical, biological, and social sciences - as long as we do not become involved in quantum mechanics. (1984, p. 278)

According to Salmon, explaining ...

... involves the placing of the explanandum in a causal network consisting of relevant causal interactions that occurred previously and suitable causal processes that connect them to the fact-to-be-explained. (1984, p. 269)

The usual label for explanations of this type is "causal-mechanical explanation" (abbreviated here as CM explanation).

These quotes clearly reveal a descriptive aim, which is captured in the following hypothesis:

All scientists (except maybe in quantum mechanics) who have understanding as an aim really seek CM explanations.

The aim of Salmon's book is to clarify what CM explanations are and to argue for this claim. Salmon only succeeds in the first task: he defines the crucial concepts, viz. causal interaction and causal process. Arguments for the descriptive claim are missing: there is only the hope to have succeeded (cf. the quote above, which comes from the very end of the book). Like Hempel and Kitcher, Salmon did not try to collect empirical evidence. ${ }^{2}$

\section{Summary and lessons}

Hempel, Kitcher and Salmon not only developed explications, they also did something with them. All three of them made descriptive claims, Hempel and Kitcher made normative claims.

When a philosopher (or a scientist) makes a descriptive claim, it is legitimate to expect some kind of empirical evidence to back up this claim. Hempel, Kitcher and Salmon do not live up to this expectation. They decided to make descriptive claims, so they should have provided evidence. But they did not. In order to close that evidential gap, we can start collecting evidence. However, the task is huge. Therefore, it may be a good idea to make less general descriptive claims: more modest claims require less evidence. This is one of the motivations for our pragmatic approach. This idea will be elaborated in Section 6 .

When a philosopher puts forward a normative claim, it is legitimate to expect some kind of argument for it. Hempel and Kitcher do not live up to this expectation. They decided to make normative claims, so they should have provided arguments. But they did not. If we want to do better,

\footnotetext{
${ }^{2}$ Salmon does not make any normative claims.
} 
we should take a step back and ask ourselves: how can we argue for such normative claims about explanation and understanding? This is what we do in the pragmatic approach, by means of the concept of epistemic interest (see below). And like in the descriptive case, it may be wise to make less general claims; as explained below this is also an ingredient of the pragmatic approach.

\section{A pragmatic approach to studying scientific explanations: the basic ideas}

\section{Preliminary remarks}

"Pragmatic approach to scientific explanation" is a label we started using a couple of years ago to denote the way we have been studying scientific explanations since roughly the year 2000 . This means that we do not claim that we present something "brand new" here (something that has not been tried before). We have done this ourselves for more than a decade. Nor do we claim exclusivity: other philosophers of explanation have taken a similar approach (see the examples at the end of Section 7). However, as far as we know, this paper is the first attempt to reflect on a meta-level on what has changed. Our motivation for writing this paper is that not all change is automatically progress. Therefore, it makes sense to try to characterise our own practice and that of like-minded philosophers of explanation and try to find out whether and - if so - how it is better than what Hempel, Kitcher and Salmon did. The first step in characterising what is going on was to choose a label "pragmatic approach to scientific explanation". Let us now clarify what it consists in by discussing what its results are: context-specific normative claims and context-specific descriptive claims.

\section{Context-specific normative claims}

The first guiding principle of the pragmatic approach is: make context-specific normative claims and argue for them. This means that you first look at how scientists actually explain in certain disciplines and within certain research traditions in a discipline and then reflect upon it. This reflection can have three forms. We can defend certain explanatory practices (e.g. when other scientists in the discipline deny the validity or usefulness of that practice) or criticise explanatory practices. And we can also try to provide guidelines for improving an explanatory practice. Examples of this can be found in Van Bouwel \& Weber (2008a) and (2008b) and in De Vreese, Weber \& Van Bouwel (2010). These papers engage in debates on explanatory practices in the social sciences and in the biomedical sciences. Models of scientific explanation as developed by philosophers of science are used as tools to make context-specific normative claims which reflect on (i.e. criticise, defend and/or try to improve) actual explanatory practices.

The fact that we use traditional models of explanation as toolbox is one of the reasons why we call our approach pragmatic (there are more reasons, see below). "Pragmatic" thus refers to an "instrumentalist" attitude towards models of explanation: we see them as tools in a toolbox, rather than as describing the "essence" of explanation or understanding. In doing this, we differ in one important respect from the normative endeavours of Hempel, Kitcher and Salmon: the claims we make are context-specific, i.e. they relate to specific explanatory practices of specific scientists or groups of scientists. There are (at least) two types of normative claims: exclusion rules and preference rules. Suppose someone claims that "DN explanations that do not describe causes are always useless". That is an exclusion rule (it claims that a certain type of explanations is not useful and thus excludes them). Moreover, it is a very general exclusion rule, because of the "always". What we propose, among other things, is to make more modest exclusion claims, for instance: "In this context, it is useless to construct a DN explanation which does not describe causes." Analogously, we propose to formulate context-specific preference rules, for instance "In this context, 
a CM explanation is better than an DN explanation". As this example illustrates, preference rules tell us which of two types of explanation is the best if both types are available. We propose to make context-specific preference claims, not general ones. An example of a general one would be "CM explanations are always better than DN explanations".

Our examples of exclusion and preference rules make clear how we use the term "context": it is used to limit the intended scope of normative claims (it will be used in the same way for descriptive claims below). It is difficult to give a more precise characterisation of what a context is, because we have to allow for variation in size: it may be one scientist dealing with one problem, a small group of scientists dealing with related problems, a subdomain of a scientific discipline, ....

There are several reasons for why we propose the piecemeal approach in which the normative claims have a limited scope. First, this approach remains close to the actual practice of scientists in the relevant disciplines and, therefore, it will be easy for the scientists to connect the results to their practice (which increases the chance that philosophical reflection has an impact on scientific practice). Second, one possible way to arrive at general normative claims is bottom-up (i.e. by generalising from context-specific claims, if they turn out to be similar). So adopting a piecemeal approach is heuristically useful for someone who has a more ambitious aim. Third (and related to the second reason), the context-specific claims are more modest and therefore normally easier to argue for.

Another difference is that we not only put forward normative claims; we also argue for them in our papers. In these arguments we use epistemic interests as our main tool. Epistemic interests are types of motives for (a) scientists to search for explanations and (b) other people (policy makers, the general public) to be interested in the explanations scientists give and to pay them for their research. Examples of epistemic interests are improvement (i.e. the desire of making a given situation better) and prevention (which is closely related to improvement, but more future-oriented: avoiding undesirable situations). A third example is attributing moral and/or legal responsibility: this is often the underlying epistemic interest when we explain actions (see Weber \& Vanderbeeken 2005 for this) or disasters (see our case study in Section 8). The epistemic interests which motivate the search for an explanation influence the type of explanation that is appropriate in a given case and also influence which properties of an explanation (e.g. depth, deductivity) are important and which not in the case. For this reason, epistemic interests are a good tool to argue for normative claims. In the case study in Section 8, we will clarify how this works. ${ }^{3}$ The use of epistemic interests constitutes a second reason to call our approach pragmatic: the idea of epistemic interests originates in the work of the American pragmatists (Charles Peirce, John Dewey) and is also clearly present in the work of the German pragmatist Jürgen Habermas (who uses the term "knowledge-constitutive interest", see Habermas 1978).

\section{Context-specific descriptive claims}

The second principle is: try to make context-specific descriptive claims and argue for them. This means that you try to describe the explanatory practice of scientists in a certain discipline or research tradition. For instance, Walmsley (2008) describes the explanatory practice of dynamical cognitive scientists and argues that they are constructing DN explanations (in Gervais \& Weber (2011) it is nonetheless shown that this description is inaccurate). In these descriptions, traditional models of explanation are again used as tools in a toolbox (this is a third reason to call the approach pragmatic).

The advantage of this piecemeal approach (as compared to the general claims made by Hempel, Kitcher and Salmon) is that we do not need large samples of scientists we can interview or large samples of scientific writings we can analyse. In Section 8 we will use Richard Feynman's

\footnotetext{
${ }^{3}$ The relation between epistemic interests, types of explanation and the value of properties of explanations is investigated in Weber, Van Bouwel \& Vanderbeeken (2005) and Weber \& Van Bouwel (2007).
} 
investigation of the Challenger disaster as an example. Our aim there is to find out what kind of explanations Feynman was giving in his report. That aim is very modest compared to the bold general claims that Hempel, Kitcher and Salmon make. The advantage is that we only have to look at Feynman's report, we do not have to care about other scientists in the world (nor about Feynman's other work). Another illustration is the following. Suppose we want to describe how population geneticists explain micro-evolutions in populations. That is not a trivial task (for instance, we have to analyse all important textbooks on population genetics before we can make a claim that is relatively well supported) but at least we don't have to worry about what other biologists do or about what physicists, psychologists, sociologists, etc. do. The general claims that Hempel, Kitcher and Salmon make, require that we take into account all scientists in the world. That is logically possible, but not feasible at this moment. So at least for now we have to settle for the more modest descriptive endeavours we are proposing.

\section{A pragmatic approach to studying scientific explanations: some comparisons}

To clarify our approach further, we make four comparisons.

\section{Methodological neutrality versus methodological commitment}

Let us go back to Carnap's idea of explication. The pragmatic approach we defend still assumes that we develop explicata as a first step in the analysis of explanations: we propose to use them - in the same way as Hempel, Kitcher and Salmon did - to put forward descriptive and normative claims. The reason is that without explicata we have no precise claims. And if the meaning of the claims remains vague, we cannot develop decent arguments. So if we want to develop arguments, we need explicata. However, our approach requires that we give up an implicit assumption which Carnap makes. Carnap seems to assume that philosophers of science can get far by developing one explicatum for a given explicandum (e.g. probability or explanation). Hempel, Kitcher and Salmon certainly assume this: they are convinced that their explicatum will do all the work (maybe leaving out some "strange" fields like QM, cf. Salmon). Our pragmatic approach does not presuppose this monism and adopts pluralism as a heuristic guideline: in principle, every explicatum that has been developed by a philosopher of explanation in the past can be used to formulate a context-dependent descriptive or normative hypothesis that is then further investigated. In the end - after analysing all explanatory practices of scientists - it may turn out that all explicata except one are superfluous. However, an a priori choice for monism is an overhasty conclusion which is unfounded at this moment and thus - from a methodological point of view - a bad start for studying scientific explanation.

A different way of putting this is that our pragmatic approach is methodologically neutral: we use whatever is available (all the explicata that have been developed) as tools for analysing explanatory practices (both descriptively and normatively) and see what works best in a given context. Hempel, Kitcher and Salmon were methodologically committed: they first put a lot of effort in developing their models; and then of course they wanted to show that their models were good or better than the models of their rivals. So they were committed to showing that their efforts in developing an explicatum were not in vain. We do not have such an agenda, because we are eclectic with respect to existing models. Therefore nothing can distract us from our main aim: making context-specific normative and descriptive claims and argue for them. This is an important advantage of our methodological neutrality. 
Pragmatic approach versus pragmatic theory

Our pragmatic approach to explanations should not be confused with a pragmatic theory of explanation as developed by e.g. Bas van Fraassen (1980). According to a pragmatic (also known as erotetic) theory of explanation, an explanation is an answer to a why-question that uses the appropriate relevance relation. Which relevance relation is appropriate depends on the context. Though this theory is much vaguer than the model of e.g. Hempel, Kitcher and Salmon, it is still a theory about what all explanations have in common. Our proposal - the pragmatic approach - is situated at the meta-level: it is a proposal on how to study scientific explanation. One of the ingredients of the approach - as outlined above - is that we use models of explanations as instruments. So we stop making general claims about the nature of all scientific explanations. We do not even make vague claims like in the pragmatic theory of van Fraassen. We give up generality so that we can make precise, informative claims (which are valid in specific contexts). Van Fraassen goes in the opposite direction: he wants to retain the generality and therefore ends up with vague claims.

\section{Examples of philosophers of explanation with a similar (but not identical) approach}

As mentioned at the beginning of Section 6, we do not claim exclusivity. In the Preface of his book Explaining the Brain Carl Craver says that his project is both descriptive and normative. On the descriptive side, he claims that "[t] he goal of searching for mechanistic explanations is now woven through the fabric of neuroscience[.]" (2007, p. viii) On the normative side, he wants to find out "what is required of an adequate explanation in neuroscience?" ( $p$. vii). Craver's claims are clearly context-specific, both the descriptive and the normative ones: their intended scope is the domain of neuroscience. With respect to aims and scope, his approach fits into what we propose. However, in the way he proceeds he is committed to the mechanistic model of explanation which he develops. $\mathrm{He}$ is not methodologically neutral in the way we are. Though he does not deny that other types of explanation may be important for neuroscience, it is clear that for Craver one can get very far in the analysis of neuroscientific explanations by means of only one tool (the mechanistic model).

A second example is Daniel Little's book Varieties of Social Explanation (1991). The aim of this book is to get grip on the nature of explanation in the social sciences. This is clearly context-specific. In Chapter 1 Little presents some philosophical models of explanation (Hempel's DN and IS models and Salmon's statistical relevance model) which he intends to use as tools. Throughout the book, more tools are added (e.g. a model of functional explanations in Chapter 5). So Little is methodological neutral. The main difference with our approach is that we would plead for a more extensive and systematic presentation and application of philosophical models of explanation. Neither is the potential of the philosophical literature on models of explanation fully used in Little's book nor does he develop a metaphilosophical framework for dealing with the plurality of models of explanation.

\section{Some old fashioned philosophers of explanation}

Jim Woodward's book Making Things Happen. A Theory of Causal Explanation is about causation and explanation. We only look at explanation here. His project is both descriptive and normative (2003, pp. 7-8). He restricts himself to "causal explanations broadly conceived". According to this broad notion of causal explanation "any explanation that proceeds by showing how an outcome depends (where the dependence in question is not logical or conceptual) on other variables counts as causal" (p. 6). The claims he makes about these explanations are certainly not context-specific. The core idea of the counterfactual theory which he presents in Chapter 5 of the book is that "to explain an outcome, one must provide information about the conditions under which it would change or be different" (p. 233). This is supposed to be valid in all scientific domains and in all contexts. In Chapter 8 of the book, Woodward compares his theory with what he considers its most important rivals: 
Kitcher's unification account and Salmon's causal mechanical model. This means that he sees himself as doing the same type of project, which is correct.

It goes without saying that we think that Woodward's project also failed. On the one hand he does not meet the requirements of providing sufficient empirical evidence and providing good arguments for his prescriptions. On the other hand, it is quite easy to find counterexamples to both the general descriptive and normative claim he makes (see our case study in Chapter 8 for this).

A second example is Depth. An Account of Scientific Explanation by Michael Strevens. Strevens agrees that in principle the philosophical study of scientific explanation can be descriptive or evaluative. However, he almost exclusively wants to focus on description (2008, p. 37). Here is a quote that illustrates what he is after and at the same time points at a serious problem:

The goal of the descriptive project is to say what kinds of explanations we give and why we give them. This is what I call our explanatory practice. The most important source of evidence concerning our explanatory practice is the sum total of the explanations regarded as scientifically adequate in their day, together with an understanding of the background against which they seemed adequate. $(2008$, p. 37)

This quote makes clear that Strevens' descriptive project is as ambitious as the projects of Hempel, Kitcher and Salmon. It also contains the clue as to why it is doomed to fail: Strevens does not have the means to gather the evidence he is after ("the sum total of explanations regarded as scientifically adequate").

The fact that the projects of Woodward and Strevens are problematic does not imply that their ideas are useless. On the contrary, they can be integrated into a pragmatic approach. Woodward's desideratum that explanations must provide answers to what-if-things-had-been-different-questions is, in our view, valid if we are dealing with a contrastive explanation-seeking question. So we would make context -specific evaluative claims with it, but deny it the overall status that Woodward assigns to it. This will be illustrated in the case study in Section 8.

The same is true for Strevens' "optimising procedure", which is the core of his kairetic theory of explanation. Abstraction vs. level of detail is an important dimension in the evaluation of explanatory practices: an explanation should not contain irrelevant details, but should not leave out relevant information either. Strevens' optimising procedure is meant to achieve this. For a proponent of our pragmatic approach, this is a useful instrument for justifying context-specific claims. But we would drop the overall, very general project which Strevens entertains.

\section{A case study: Richard Feynman on the Challenger disaster}

\section{Introduction}

The Challenger disaster happened at Kennedy Space Center in Florida on January 28, $1986 .{ }^{4}$ Space shuttle Challenger lifted off at 11:38:00 a.m. (Eastern Standard Time) and exploded 73 seconds later. The entire crew (seven astronauts) was killed. To investigate the causes of the accident, president Reagan appointed a presidential commission. It was headed by former secretary of state William Rogers and included, among others, former astronaut Neil Armstrong and Nobel Prize winning physicist Richard Feynman.

Feynman tells about this episode in his life in one of his books (Feynman 1988). Feynman and the commission as a whole focussed on two questions. The first was: what physically caused the

\footnotetext{
${ }^{4}$ There are various online resources which describe what happened on that day. We use the texts of Nick Greene in About.com.
} 
explosion of the Challenger? The second question was: what went wrong in NASA that made the explosion possible? The underlying epistemic interest for asking the second question is obvious: prevention of similar disasters. The first question is useful because answering it may help us to refine the second question (see below). However, it can also be useful for determining responsibility.

Before we have a closer look at Feynman's search for information and the resulting explanations, it is useful to give some background knowledge. The fatal mission (its official name was mission 51-L) was in several respects a standard mission. Challenger was not the first operational space shuttle (Columbia was the first one) and it had done nine successful missions, so there was nothing exceptional in those respects. Also, the cargo was the usual stuff (a satellite and scientific apparatus). However, the mission was exceptional in two respects. First, one of the crew members was Christa MacAuliffe, who was supposed to become the first school teacher in space. This caused large media coverage of the accident. Second, it was a very cold day. This turned out to be important, as we will see below.

\section{A causal-mechanical explanation}

To find an answer to the first question, Feynman obtained technical information, primarily on the working of the solid rocket boosters (SRBs), the space shuttle main engines (SSMEs) and the flight electronics (see Feynman 1988 for details). Very early in his search for answers, it became clear to Feynman that there was a serious problem with the rubber O-rings that should seal the aft field joint of the right SRB. SRB's are built up from different smaller segments that are connected by a Tang and Clevis joint: each segment has a U-like shape at the top, such that the bottom of the segment above can nicely slide into it. To prevent that the gases inside the SRB can leak through these joints, they are sealed with two rubber O-rings that are supposed to be very flexible. However, it turned out that it was not clear how these rubber seals would react to the extremely cold temperature on the morning of the launch, and that some technicians had already expressed their fear for problems resulting from the O-rings beforehand.

What Feynman actually did to get an answer to his first question, was searching for an acceptable causal-mechanical explanation which described the detailed technical process that led to the explosion. He investigated what technicians knew about the behaviour of the specific parts of the space shuttle's rocket boosters and main engines, and about the influence of the specific conditions under which these mechanisms had to work on the specific morning of the Challenger's launch, namely the extreme cold. These brought him to an explanation showing how gases could leak, causing a fire blowing up the space shuttle. Nick Greene summarises this explanation as follows:

The commission's report cited the cause of the disaster as the failure of an "O-ring" seal in the solid-fuel rocket on the Space Shuttle Challenger's right side. The faulty design of the seal coupled with the unusually cold weather, let hot gases to leak through the joint. Booster rocket flames were able to pass through the failed seal enlarging the small hole. These flames then burned through the Space Shuttle Challenger's external fuel tank and through one of the supports that attached the booster to the side of the tank. That booster broke loose and collided with the tank, piercing the tank's side. Liquid hydrogen and liquid oxygen fuels from the tank and booster mixed and ignited, causing the Space Shuttle Challenger to tear apart. (Greene (B)) 


\section{A counterfactual explanation}

This causal-mechanical explanation, although giving us good insight in what caused the explosion of the Challenger, does not answer the second explanation-seeking question that Feynman and the committee addressed. However, it enabled them to formulate two follow-up questions:

Why was the space shuttle program continued, rather than put on hold till the problems of gas leakage and erosion were solved?

Why was the decision taken to launch the Challenger on January 28th, instead of postponing the launch to a less cold day?

These questions are more specific than the original second question (What went wrong within NASA?) They presuppose knowledge of what physically caused the explosion, so we cannot formulate these questions without answering he first question. The answers which Feynman and the presidential committee give are counterfactual in the sense proposed by Woodward: they tell us under which conditions the effect would have been different. They first describe how NASA really functioned and then tell us how things should have been different in order to obtain the alternative (more desirable) fact. We focus on the first follow-up question.

During some previous missions there had been gas leakages in some seals, resulting in clearly visible black marks on the rocket (at places where hot gas had leaked) and so-called 'erosion' (spots where the $\mathrm{O}$ rings were partially burnt). These two problems show that the O-rings did not perform their function (viz. sealing) perfectly. This shortcoming is the "faulty design" to which Greene refers in the quote above. According to Feynman, the way in which NASA officials dealt with these incidents was completely wrong. He compares their risk analysis methods and risk management strategies to Russian roulette. Here is what Feynman writes:

The phenomenon of accepting for flight, seals that had shown erosion and blow-by in previous flights, is very clear. The Challenger flight is an excellent example. There are several references to flights that had gone before. The acceptance and success of these flights is taken as evidence of safety. But erosion and blow-by are not what the design expected. They are warnings that something is wrong. The equipment is not operating as expected, and therefore there is a danger that it can operate with even wider deviations in this unexpected and not thoroughly understood way. The fact that this danger did not lead to a catastrophe before is no guarantee that it will not the next time, unless it is completely understood. When playing Russian roulette the fact that the first shot got off safely is little comfort for the next. The origin and consequences of the erosion and blow-by were not understood. They did not occur equally on all flights and all joints; sometimes more, and sometimes less. Why not sometime, when whatever conditions determined it were right, still more leading to catastrophe?

In spite of these variations from case to case, officials behaved as if they understood it, giving apparently logical arguments to each other often depending on the "success" of previous flights. For example, in determining if flight 51-L was safe to fly in the face of ring erosion in flight 51-C, it was noted that the erosion depth was only one-third of the radius. It had been noted in an experiment cutting the ring that cutting it as deep as one radius was necessary before the ring failed. Instead of being very concerned that variations of poorly understood conditions might reasonably create a deeper erosion this time, it was asserted, there was "a safety factor of three." This is a strange use of the engineer's term, "safety factor." If a bridge is built to withstand a certain load without the beams permanently deforming, cracking, or breaking, it may be designed for the materials used to actually stand up under three times the load. This "safety factor" is to allow for uncertain excesses of load, or unknown extra loads, or 
weaknesses in the material that might have unexpected flaws, etc. If now the expected load comes on to the new bridge and a crack appears in a beam, this is a failure of the design. There was no safety factor at all; even though the bridge did not actually collapse because the crack went only one-third of the way through the beam. The O-rings of the Solid Rocket Boosters were not designed to erode. Erosion was a clue that something was wrong. Erosion was not something from which safety can be inferred.

There was no way, without full understanding, that one could have confidence that conditions the next time might not produce erosion three times more severe than the time before. Nevertheless, officials fooled themselves into thinking they had such understanding and confidence, in spite of the peculiar variations from case to case. (Feynman 1986; italics added)

This quote nicely summarizes the descriptive part of Feynman's explanation (there are much more details about specific decisions and measurements in other parts of the Commission report). The sentences we put in italics point at the different assessment procedure, which in Feynman's view, would have led to the alternative, desirable result: NASA officials should have interpreted the gas leakages and erosions as warning signs, as indications that something was wrong. If they would have done that, they would have put the space shuttle programme on hold.

\section{Comments on the case study}

This case study illustrates several aspects of the pragmatic approach:

(1) We only investigate the report of Feynman and his colleagues. The evidence one has to consult for that is clear and limited: the reports they have published on the Challenger disaster. This makes the descriptive task feasible: we can discern and describe two explanatory questions that motivated Feynman's research. Furthermore, we can see that the questions were answered by means of a different kind of explanation (causal-mechanical versus counterfactual).

(2) The epistemic interests which are involved are clear, and the case nicely illustrates how epistemic interests lead to desiderata in the appropriate format of the explanation, and thus can be used to argue for normative claims about explanations. For instance, the epistemic interest that motivates the second question is more adequately addressed by a counterfactual explanation, while a causal-mechanical explanation would answer the question in a less adequate and less efficient way, given the interests of the explainers, i.e. scientists, or the explainees, i.e. other people (policy makers, the general public) looking for answers to their specific explanation-seeking questions.

\section{Conclusion}

In this paper, we have presented the descriptive and normative claims on scientific explanation that Hempel, Kitcher and Salmon make by means of their explicata. We have identified a major shortcoming which they have in common: they do not gather empirical evidence for their descriptive hypotheses (this could be done by systematically investigating the writings of a large representative sample of scientists) and do not argue for their normative claims either.

We presented our pragmatic approach to studying scientific explanation as a better way to proceed. The main differences of what we propose - compared to Hempel, Kitcher and Salmon - are: context-specificity of the normative and descriptive claims, providing arguments instead of merely putting forward hypotheses, and the role of epistemic interests. We hope that the theoretical arguments in Section 6, the comparisons in Section 7 and the case study in Section 8 suffice to make our ideas clear and to show that the approach is worthwhile. Readers that are not convinced can probably only be convinced by more case studies. These can be found in the papers mentioned 
before and in Weber, Van Bouwel \& De Vreese (2013).

\section{References}

Carnap Rudolf (1950), Logical Foundation of Probability. London: Routledge and Keegan Paul.

Cartwright Nancy (1983), How the laws of physics lie. Oxford: Clarendon Press.

Craver Carl (2007), Explaining the Brain. Oxford: Clarendon Press.

De Vreese Leen, Weber Erik \& Van Bouwel Jeroen (2010), 'Explanatory Pluralism in the Medical Sciences: Theory and Practice', in Theoretical Medicine \& Bioethics 31, pp. 371-390.

Feynman Richard (1986), 'Appendix F- Personal Observations of the Reliability of the Shuttle', http://history.nasa.gov/rogersrep/v2appf.htm. Accessed 30 August 2012. [Reprinted in Feynman 1988].

Feynman Richard (1988), What Do You Care What Other People Think? New York \& London: W. W. Norton.

Gervais Raoul \& Weber Erik (2011), 'The Covering Law Model Applied to Dynamical Cognitive Science: a Comment on Joel Walmsley', in Minds and Machines 21, pp 33-39.

Greene Nick (A), 'Challenger Disaster - a NASA Tragedy. Part 1: The Launch and Disaster' in About.Com (Part of the New York Times Company). Http://space.about.com/cs/challenger/a/challenger.htm. Accessed 30 August 2012.

Greene Nick (B), 'Space Shuttle Challenger Disaster - a NASA Tragedy. Part 2: The Space Shuttle Challenger Aftermath' in About.Com (Part of the New York Times Company). Http://space.about.com/cs/challenger/a/challenger 2.htm. Accessed 30 August 2012.

Habermas Jürgen (1978), Knowledge and Human Interests. Second Edition. London: Heinemann Educational Books. [Translation of Erkenntnis und Interesse. Frankfurt: Suhrkamp Verlag, 1968].

Hempel Carl (1965), Aspects of Scientific Explanation and other Essays in the Philosophy of Science. New York: Free Press.

Hempel Carl \& Oppenheim Paul (1948), 'Studies in the Logic of Explanation', in Hempel (1965), pp. 245-290. [originally in: Philosophy of Science 15)

Humphreys Paul (1989), The Chances of Explanation. Princeton, NY: Princeton University Press.

Kitcher Philip (1981), 'Explanatory Unification', in: Philosophy of Science 48, pp. 507-531.

Kitcher Philip (1989), 'Explanatory Unification and the Causal Structure of the World', in: Kitcher \& Salmon (1989), pp. 410-505.

Kitcher Philip \& Salmon Wesley (eds.) (1989), Scientific Explanation. Minneapolis: University of Minnesota Press.

Knobe Joshua (2004), 'What Is Experimental Philosophy?', in The Philosophers' Magazine 28.

Little Daniel (1991), Varieties of Social Explanation. Boulder, CO: Westview Press.

Salmon Wesley (1984), Scientific Explanation and the Causal Structure of the World. Princeton, NY: Princeton University Press.

Salmon Wesley (1989), 'Four Decades of Scientific Explanation', in: Kitcher \& Salmon (1989), pp. 3-219.

Strevens Michael (2008), Depth. An Account of Scientific Explanation. Cambridge: Harvard University Press.

Van Bouwel Jeroen \& Weber Erik (2008a), 'De-ontologizing the Debate on Social Explanations: a Pragmatic Approach Based on Epistemic Interests', in Human Studies 31, pp. 423-442.

Van Bouwel Jeroen \& Weber Erik (2008b), 'A pragmatic defence of non-relativistic explanatory pluralism in history and social science', in History and Theory 47, pp. 168-182.

van Fraassen Bas (1980), The Scientific Image. Oxford: Oxford University Press. 
Walmsley Joel (2008), 'Explanation in Dynamical Cognitive Science', in Minds and Machines 18, pp. 331-348.

Weber Erik, Van Bouwel Jeroen \& Vanderbeeken Robrecht (2005), 'Forms of Causal Explanation', in Foundations of Science 10, pp. 437-454.

Weber Erik \& Van Bouwel Jeroen (2007), 'Assessing the Explanatory Power of Causal Explanations', in Johannes Persson \& Petri Ylikoski (eds.), Rethinking Explanation. Dordrecht: Kluwer Academic Publishers/Springer, pp. 109-118.

Weber Erik, Van Bouwel Jeroen \& De Vreese Leen (2013), Scientific Explanation. Dordrecht: Springer. Weber Erik \& Vanderbeeken Robrecht (2005), 'The Functions of Intentional Explanations of Actions', in Behavior and Philosophy 33, pp. 1-16.

Woodward James (2003), Making Things Happen. A Theory of Causal Explanation. New York: Oxford University Press. 


\section{Appendix: Hempel's covering law model and its problems}

The DN model

We start with some definitions and terminology. According to Hempel, an explanation consists of an explanandum $E$ (a description of the phenomenon to be explained) and an explanans (the statements that do the explaining). He distinguishes between true explanations and explanations that are well-confirmed (see e.g. Hempel 1965b, p. 338). Both concepts are defined by means of the auxiliary concept of potential explanans, which is characterized as follows (cf. Hempel \& Oppenheim 1948, part 3):

(DN) The ordered couple $(L, C)$ constitutes a potential explanans for the singular sentence $E$ if and only if

(1) $L$ is a purely universal sentence and $C$ is a singular sentence,

(2) $E$ is deductively derivable from the conjunction $L \& C$, and

(3) $\mathrm{E}$ is not deductively derivable from $\mathrm{C}$ alone.

A purely universal sentence consists of one or more universal quantifiers, followed by an expression which contains no quantifiers and no individual constants. $(L, C)$ is a true explanans for $E$ if and only if $(L, C)$ is a potential explanans for $E$ and both $L$ and $C$ are true. $(L, C)$ is a well-confirmed explanans for $E$ if and only if $(L, C)$ is a potential explanans for $E$ and both $L$ and $C$ are well-confirmed. ${ }^{5}$

Let us consider an example. In volume I of The Feynman Lectures on Physics, Chapter 26 deals with elementary optics. There we find a simple law about mirrors ${ }^{6}$ :

The simplest object is a mirror, and the law for a mirror is that when the light hits the mirror, it does not continue in a straight line, but bounces off the mirror into a new straight line[.] ... The light striking a mirror travels in such a way that the two angles, between each beam and the mirror, are equal. (Feynman et al. 2010, p. 26-2)

Suppose that we have the following explanandum

E: $\quad$ This reflected beam of light $a$ has an angle of $45^{\circ}$ relative to the mirror from which it bounced.

According to definition (DN), the following construction is a potential explanans for this phenomenon:

C: $\quad$ The angle of incidence of $a$ relative to the mirror was $45^{\circ}$.

$\mathrm{L}$ : $\quad$ For all beams reflecting on mirrors: if the angle of incidence relative to the mirror is $45^{\circ}$, then the reflected beam also has an angle of $45^{\circ}$ relative to the mirror.

If all these claims are true, we have a true explanans.

This example illustrates a general property of DN explanations. The first two conditions in (DN) imply that explanations have the form of a deductive argument. The simplest format is this:

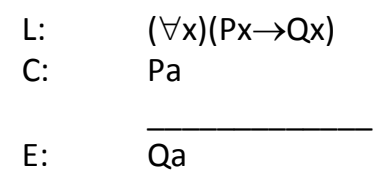

The mirror example fits this simple format. In more complex explanations, $\mathrm{C}$ is a conjunction of atomic singular sentences $\mathrm{C}_{1}, \mathrm{C}_{2}, \ldots$

The third condition in $(\mathrm{H})$ excludes circular arguments as explanations. The following deductive arguments

\footnotetext{
${ }^{5}$ An explanans may be true even if we don't have any evidence in favour of it. And a well-confirmed explanans may be false (which means that our evidence is incomplete and therefore misleading). For these reasons, Hempel introduces two concepts based on the concept of potential explanans.

${ }^{6}$ Note that Feynman assumes that the mirrors are flat.
} 
are no explanations, though they satisfy the two first conditions:

$$
\begin{array}{ll}
\text { L: } & (\forall x)(P x \wedge Q x \rightarrow R x) \\
\text { C: } & \mathrm{Pa} \wedge \mathrm{Qa} \\
\text { E: } & \mathrm{Qa} \\
\text { L: } & (\forall \mathrm{x})(\mathrm{Px} \rightarrow \mathrm{Px}) \\
\text { C: } & \mathrm{Pa} \\
\text { E: } & \mathrm{Pa}
\end{array}
$$

In both cases, $\mathrm{E}$ is derivable from $\mathrm{C}$ alone, so the argument is circular. Such circular arguments, though deductive, are not DN explanations.

The IS model

Where the DN model is meant to capture the structure of deterministic explanations, the IS model intends to cover probabilistic explanations. Let us begin with a characterization given by Hempel:

Explanations of particular facts or events by means of statistical laws thus present themselves as arguments that are inductive or probabilistic in the sense that the explanans confers upon the explanandum a more or less high degree of inductive support or of logical (inductive) probability; they will therefore be called inductive-statistical explanations; or I-S explanations. (1965, pp. 385-386)

Explanation is still linked with expectability, but in this case expectability comes in degrees. The idea of lawlike sentences thus has to be extended to account for statistical laws that have the conditional form $\operatorname{Prob}(\mathrm{G} \mid \mathrm{F})=r$, where $r$ denotes the probability that an object of the set $F$ is also a member of the set $G$. The set $F$ is called the reference class of this statistical law.

In its simplest form, an IS explanation is an argument with the following structure, analogous to DN explanations:

$$
\begin{array}{ll}
\text { (IS) L: } & \operatorname{Prob}(\mathrm{G} \mid \mathrm{F})=\mathrm{r} \\
& \mathrm{C}: \quad \mathrm{Fb} \\
\text { ============= }[\mathrm{r}] & \mathrm{E}: \quad \mathrm{Gb}
\end{array}
$$

The notation is borrowed from Hempel: the double line indicates that the argument is inductive rather than deductive, and " $[r]$ " represents the degree of inductive support that is conferred upon the conclusion by the premises. This argument explains the fact that object $b$ has property $\mathrm{G}$ by showing that this could be expected with probability $r$, given the fact that the statistical law $L$ holds, and that $b$ has property $F$. (Of course this structure can be extended to a more general schema in which the reference class of the conditional probability is determined by a conjunction of properties $F_{1} \& F_{2} \& \ldots \& F_{n}$, and in which $b$ has the properties $F_{1}, \ldots, F_{n}$.)

Not all arguments of this form are IS explanations. As in the case of DN explanations we have to rule out the possibility of circular explanations. This can be done in exactly the same way: we have to require that $E$ does not follow deductively from C. For instance, the following argument is not an IS explanation because it is circular:

$$
\begin{array}{ll}
\mathrm{L}: & \operatorname{Prob}(\mathrm{G} \mid \mathrm{G})=1 \\
\mathrm{C}: \quad \mathrm{Gb} \\
\text { ============== } \\
\mathrm{E}: \quad \mathrm{Gb}
\end{array}
$$

Another extra condition is that $r>\varepsilon$, with $\varepsilon$ a chosen minimal degree of inductive support. Hempel calls this the 
high probability requirement (HPR). He requires that $r$ is high without specifying exactly how high. However, $r$ must always be higher than 0.5 , otherwise we have an argument that makes us expect that the explanandum would not happen. In other words: the lower limit on our choice of $\varepsilon$ is 0.5 .

In order to illustrate scheme IS and the HPR, we look at two examples. The following argument is an IS explanation provided that we set the value of $\varepsilon$ at between 0.5 and 0.8 .

L: $\quad 81 \%$ of the $12-18$ year old inhabitants of Flanders has a smartphone. ${ }^{7}$

C: Jan is between 12 and 18 years old and lives in Flanders.

$=============[0,81]$

E: Jan has a smartphone.

The following construction (based on an example in Toulmin 1958, p. 111) is certainly not an IS explanation because HPR is not satisfied:

L: $\quad 2 \%$ of the inhabitants of Sweden are Roman Catholic.

C: $\quad$ Petersen is a Swede.

$=============[0,02]$

E: Petersen is a Roman Catholic

\section{Accidental generalisations}

Hempel realised that he needed to distinguish between genuine laws and accidental generalizations. Consider the following two statements, apparently equivalent as far as their logical form goes (see Salmon (1989), p. 15):

(i) No gold sphere has a mass greater than $100,000 \mathrm{~kg}$.

(ii) No enriched uranium sphere has a mass greater than $100,000 \mathrm{~kg}$.

Whereas the second statement seems to be the expression of a lawful fact (the critical mass for enriched uranium is just a few kilograms; so we cannot create such a sphere because a much lighter sphere would already explode), the truth of the first statement seems to be a contingent matter of fact (it just happens to be the case that no one did produce such a sphere as yet; there is enough gold in the world and the sphere would not explode).

Hempel's example (1965b, p. 339) is this: if we derive that Harry Smith is bald from the premises (i) that Harry Smith is a member of the Greenbury School Board for 1964, and (ii) that all members of the Greenbury School Board for 1964 are bald, this is not an explanation because we have used an accidental generalisation. Hempel's problem is that he has no viable account of how to distinguish between laws and accidental generalisations. He admits this, but does not take up the challenge.

\section{Irrelevant premises}

Several people have offered counterexamples to Hempel's models. The first group of examples are inspired by the fact that the relation of logical deduction is monotonous: if you add premises to a deductive argument, the result is still a deductive argument. This is not the case for explanations: most people will not regard arguments with superfluous premises (that otherwise satisfy Hempel's conditions) as explanations. Well known examples are:

This sample of table salt dissolves in water because I have hexed it, and all samples of hexed salt dissolve in water.

The problem is that non-hexed salt also dissolves in water.

John Jones did not get pregnant during the last year because he took birth control pills, and men who take

\footnotetext{
${ }^{7}$ Based in research done by Gent University in the Spring of 2012.
} 
birth control pills don't get pregnant.

Here the premise about the birth control pills is superfluous. This problem can be solved by adding a criterion (requirement that no premise is redundant) to Hempel's model.

\section{Asymmetry}

Several people have argued that explanation is asymmetrical. Because arguments can be reversed, this asymmetry is a problem for Hempel's models. Consider the following questions and answers:

Question 1

Why does this flagpole have a shadow of 10 metres long?

Answer 1

The flagpole is 10 metres high. The sun is at $45^{\circ}$ above the horizon.

Because light moves in a straight line, we can derive (by means of the Pythagorean Theorem) that the flagpole has a shadow of 10 metres long.

\section{Question 2}

Why is this flagpole 10 metres long?

Answer 2

The flagpole has a shadow of 10 metres long. The sun is at $45^{\circ}$ above the horizon.

Because light moves in a straight line, we can derive (by means of the Pythagorean Theorem) that the flagpole is 10 metres high.

The problem is that only the first argument is an intuitively acceptable explanation, while both answers are DN explanations in Hempel's sense.

Another famous example is the pendulum. The pendulum law $(P=2 \pi . \sqrt{ } L / g)$ describes the relation between the length of a pendulum $(L)$ and its period $(P)$. Consider the following questions and answers:

\section{Question 1}

Why does this pendulum have a period of 2.006 seconds?

Answer 1

The pendulum is 1 metre long. From this it can be derived by means of the pendulum law that its period is 2.006 seconds.

\section{Question 2}

Why does this pendulum have a length of 1 metre?

Answer 2

The pendulum has a period of 2.006 seconds. From this it can be derived by means of the pendulum law that it has a length of 1 metre.

Again we have two arguments satisfying Hempel's criteria, but only one intuitively acceptable explanation.

Hempel did not regard asymmetry as a problem: for him these examples show that we have bad intuitions about explanations. Once we realise that understanding equals expectability, we can get rid of the bad intuitions (because we realise that explanations are symmetrical). 